\title{
Influence of push-pull group substitution patterns on excited state properties of donor-acceptor co-monomers and their trimers
}

Hilde D. de Gier ${ }^{\text {a }}$, Bernd J. Rietberg ${ }^{\text {a }}$, Ria Broer ${ }^{\mathrm{a}, *}$, Remco W. A. Havenith ${ }^{\mathrm{a}, \mathrm{b}}$

a Theoretical Chemistry, Zernike Institute for Advanced Materials, University of Groningen, Nijenborgh 4, 9747 AG Groningen, The Netherlands

${ }^{\mathrm{b}}$ Ghent Quantum Chemistry Group, Department of Inorganic and Physical Chemistry, Ghent University, Krijgslaan 281, B-9000 Gent, Belgium

* Corresponding author. tel.: +31503634374

fax: +31503634441

e-mail: r.broer@rug.nl

\begin{abstract}
Organic electronics form a very promising new generation of cheap, lightweight and flexible devices. Of special interest is the ability to engineer photo-physical properties of organic molecules by chemical modification. In this regard, the purpose of this research is to understand the influence of push-pull group substitution patterns on excited state properties of several donor-acceptor comonomers an their trimers. Part of this work focuses on organic photovoltaic applications to demonstrate the practical use of the structure-property relations. In this context, the strong exciton binding energy determined by the electron-hole interaction is an important property. (Time-dependent) Density Functional Theory calculations showed a significant difference between linear- and crossconjugated push-pull group pathways for the electron-hole interaction and the vertical exciton binding energy, which can be understood from simple Hückel theory. A linear relation between the dipole moment change upon excitation and the vertical exciton binding energy hints to a possible correlation, although this relation is less pronounced for the trimers. The overlap density between the frontier molecular orbitals alone already reveals valuable information about the relative size of the electronhole interaction and the vertical exciton binding energy. Application of our findings in the context of organic photovoltaics results in significant support for cross-conjugated mesomeric push-pull group pathways in order to spatially separate the HOMO and LUMO.
\end{abstract}

\section{ARTICLE INFO}

\section{Keywords:}

donor-acceptor co-monomers and trimers inductive and mesomeric push-pull groups linear- and cross-conjugation excited state properties (time-dependent) density functional theory organic photovoltaics 


\section{Introduction}

Nowadays organic molecules find their way in all kinds of applications, like thin-film transistors (TFT), light emitting diodes (OLED) and thin-film photovoltaic cells (OPV). In comparison to conventional silicon-based electronics, organic electronics have the advantage of low-cost fabrication processes using for example roll-to-roll methods. A new generation of cheap, lightweight and flexible organic electronic devices is entering the commercial world [1]. In order to make significant progress in this field, insight in the relation between chemical structure and excited state properties is one of the key issues. Of special interest is the ability to engineer photo-physical properties by chemical modification through adding particular functional groups at different positions.

Further accelerated progress is expected by application of this knowledge in the development of new organic materials for OPV. Currently the efficiency of organic solar cells is only about $10 \%$ [2], which is limited by several factors [3]. The best-performing organic solar cell consists of a threedimensional (bulk) hetero-junction composed of a hole-conducting donor (typically a conjugated polymer) and an electron-conducting acceptor (fullerene derivative) [4]. An electronic state diagram illustrating the solar energy conversion to free charges is shown in Fig. 1 [5]. After light absorption by mainly the donor material, excitons are formed. These excitons are electron-hole pairs, bound by the Coulomb force, with an exciton binding energy $\left(\mathrm{E}_{\mathrm{b}}{ }^{\mathrm{exc}}\right)$ of $0.3-0.5 \mathrm{eV}$ [3]. They diffuse towards the donor-acceptor interface, where electrons transfer from donor to acceptor molecules. Typically, the electron-hole pair remains bound, leading to the formation of an intermolecular charge-transfer (CT) state $[3,6]$. To prevent recombination, the charge-transfer state binding energy $\left(\mathrm{E}_{\mathrm{b}}{ }^{\mathrm{CT}}\right)$ has to be overcome, e.g., with the involvement of hot CT states [5,7]. Finally, collection of free charges takes place at different electrodes of the solar cell. If the exciton binding energy can be lowered, more efficient and cost effective solar cells are within reach [3].

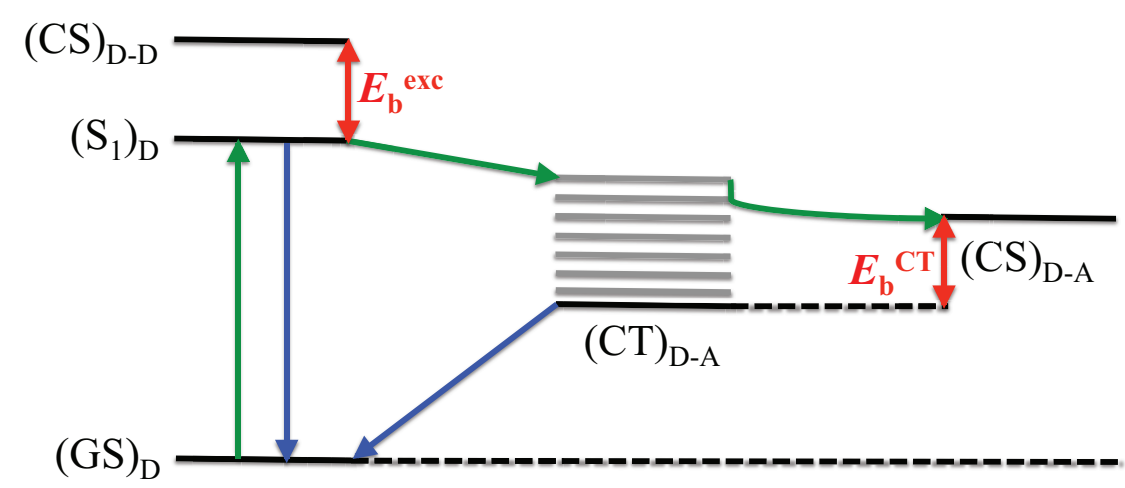

Fig. 1. Electronic state diagram describing the mechanism of converting solar energy to electric energy in organic solar cells. The green arrows show the basic steps after light absorption to create free charges when hot CT states are involved. The blue arrows show recombination pathways. The red arrows show the energy differences $\mathrm{E}_{\mathrm{b}}{ }^{\mathrm{exc}}$ and $\mathrm{E}_{\mathrm{b}}{ }^{\mathrm{CT}}$, as introduced previously. (CS) $)_{\mathrm{D}-\mathrm{D}}$ is defined as the energy difference between the ionization potential (IP) of D and electron affinity (EA) of D, and $(\mathrm{CS})_{\mathrm{D}-\mathrm{A}}$ as the energy difference between IP of D and EA of A. (GS = ground state, $\mathrm{S}_{1}=$ first excited state, $\mathrm{CS}=$ charge-separated state, $\mathrm{CT}=$ charge-transfer state, $\mathrm{D}=$ donor, $\mathrm{A}=$ acceptor)

Several experimental and theoretical studies have shown that not only Frenkel excitons, but also more separated excitons are formed by primary photo-excitations in donor polymers. These less bound excitons are believed to play a significant role in the photovoltaic process because they would increase the probability of electron transfer from donor to acceptor, decrease the energy needed for charge 
separation and reduce geminate recombination $[8,9]$. In order to distinguish between the two types of excitons, an observable can be used that correlates with their internal degree of charge separation: the change in dipole moment between the ground and excited state of the donor molecule, $\Delta \mu_{\mathrm{ge}}$ [8-10]. It was explained by Carsten et al. [9] that a larger $\Delta \mu_{\mathrm{ge}}$ correlates with a lower exciton binding energy. A relation was found between the device efficiency and the change in dipole moment upon excitation of several donor-acceptor co-monomers: when $\Delta \mu_{\mathrm{ge}}$ is larger, the efficiency increases [8]. Still, this and other correlations between solar cell performance and intrinsic properties of the donor polymers, like chemical structure, and their possible relation to the exciton binding energy of the primary photoexcitation, are not completely understood $[10,11]$.

In this regard, the objective of this work is to study the influence of push-pull group substitution patterns on excited state properties, in particular the exciton binding energy, of several donoracceptor co-monomers and their trimers. In this way we are able to understand and engineer the photo-physical properties of new molecular structures. Part of this work focuses on OPV applications to demonstrate practical use of the structure-property relations. This knowledge may be used to formulate design guides for developing new organic materials with lower exciton binding energies.

We concentrated on understanding the photo-physical properties of a donor-acceptor co-monomer (1 in Fig. 2). Monomer 1 is 4,4'-bis-(2-methyl)-4H-cyclopenta[2,1-b;3,4-b']-dithiophene-4-(2-thienyl)2,1,3-benzothiadiazole (CPDTTBT), which consists of the donating fragment CPDT and the accepting fragment BT, linked via one thiophene spacer $\mathrm{T}$. The main reason for applying donor-acceptor copolymers in organic solar cells is their low band gap enabling the absorption of more of the incoming light [12].

We studied the effect of adding $\mathrm{NH}_{2}$ as electron donating group (EDG) and $\mathrm{NO}_{2}$ as electron withdrawing group (EWG) to respectively the donor and acceptor side of this co-monomer. These groups exert mesomeric (by resonance, i.e., through $\pi$-bonds) effects. Linear- and cross-conjugated substitution patterns were compared to study their effect on excited state properties ( 2 and $\mathbf{3}$ linearand 4 and $\mathbf{5}$ cross-conjugated, Fig. 2). It is known that cross-conjugated groups show weaker electronic communication than linear-conjugated ones [13].

We investigated one conformation with $\mathrm{CH}_{3}$ as EDG and $\mathrm{F}$ as EWG added to respectively the donor and acceptor side of the co-monomer (6, Fig. 2) to get insight in the influence of inductive (i.e, through the $\sigma$-framework) effects on excited state properties. $\mathrm{NH}_{2}$ is inductively electron withdrawing and by resonance electron donating, however resonance dominates induction. $\mathrm{NO}_{2}$ is electron withdrawing both by induction and resonance, so here resonance reinforces induction. $\mathrm{CH}_{3}$ is inductively electron donating, while $\mathrm{F}$ is inductively electron withdrawing and by resonance electron donating. Here induction outcompetes resonance, so F is a weak electron acceptor [14].

For all monomers, the effect of trimerization on their excited state properties was studied to see if the trends valid for the monomers still apply. Trimerization of the reference monomer 1 and the 5 substituted monomers led to the molecules 7-12 (reference trimer 7 and 8-12, Fig. 3).

We focused on the following excited state properties: first excited state energy, oscillator strength, HOMO energy, LUMO energy, orbital energy difference between HOMO and LUMO, vertical ionization potential, and vertical electron affinity. Properties relevant for OPV applications were: change in dipole moment between the ground and excited state of the donor molecule $\Delta \mu_{\text {ge }}$, vertical exciton binding energy, the electron-hole interaction as defined in the Hartree-Fock method, and the degree of spatial overlap between occupied and virtual orbitals involved in the excitation.

This paper is organized as follows: the computational methods used in this work are described in the next section; section 3 contains the results and discussion; section 4 gives a summary of the most important conclusions. 


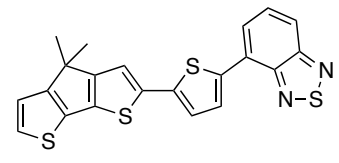<smiles>CC1(C)c2cc(-c3ccc(-c4cc([N+](=O)[O-])cc5nsnc45)s3)sc2-c2scc(N)c21</smiles>

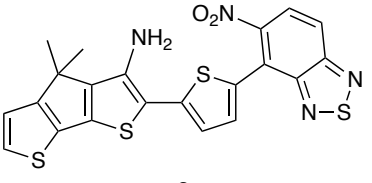<smiles>CC1(C)c2ccsc2-c2sc(-c3sc(-c4cc([N+](=O)[O-])cc5nsnc45)cc3N)cc21</smiles>
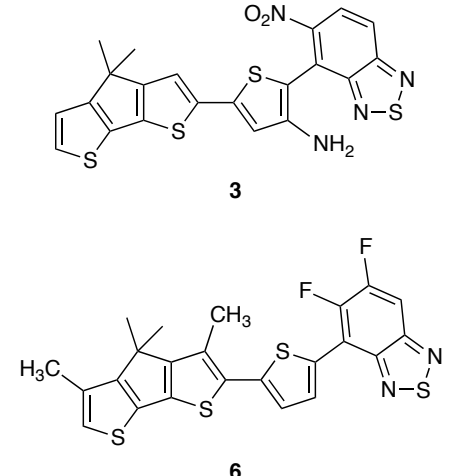

Fig. 2. Chemical structures of monomers 1-6. Molecule 1 is the reference monomer CPDTTBT. Conformations $\mathbf{2}$ and $\mathbf{3}$ are linear-conjugated and $\mathbf{4}$ and $\mathbf{5}$ are cross-conjugated. Monomers 2-5 contain mesomeric groups and monomer $\mathbf{6}$ contains inductive groups.
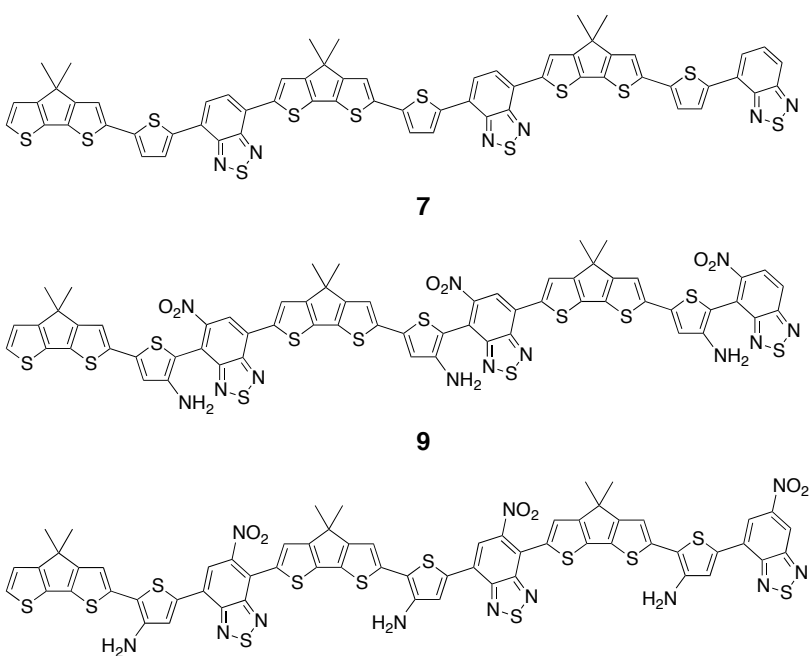

11

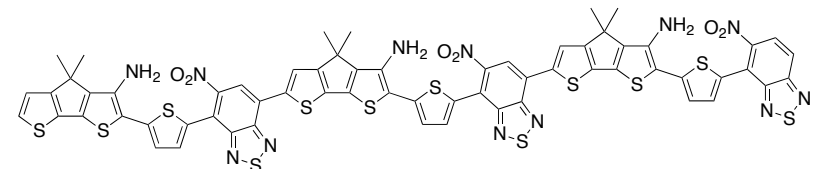

8

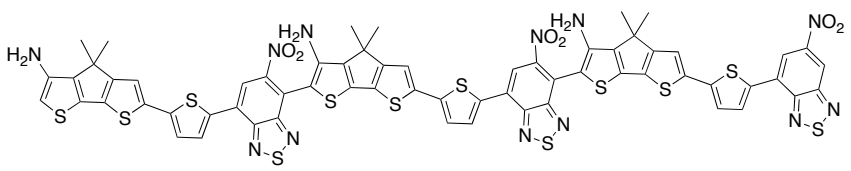

10

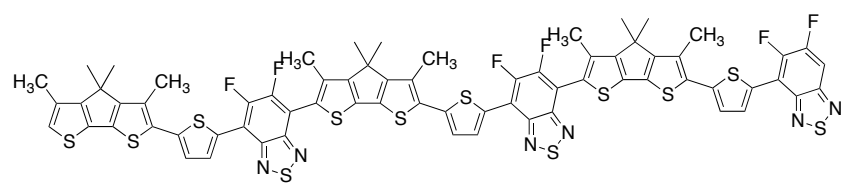

12

Fig. 3. Chemical structures of trimers 7-12. Molecule 7 is the reference trimer PCPDTTBT. Trimers 8$\mathbf{1 1}$ contain mesomeric groups and trimer $\mathbf{1 2}$ contains inductive groups.

\section{Computational methods}

Geometries of 1-12 were optimised using Density Functional Theory (DFT) (BHandH [15]/6$31 \mathrm{G}^{* *}$ ) with the programs DALTON [16] for isolated monomers 1-6 and GAMESS-UK [17] for isolated trimers 7-12. Subsequently, vertical excitation energies were calculated using time-dependent DFT (TD-DFT) (BHandH/6-31G**) with DALTON. The lowest 10 vertical excited states were calculated for isolated molecules 1-12. For all systems, the primary photo-excitation (i.e., the one with the largest oscillator strength) involved a hole predominantly located on the donating fragment and an electron on the accepting fragment. In all cases, this was the first excited state $\mathrm{S}_{1}$ for which the largest contribution comes from a HOMO to LUMO transition on the monomer or trimer.

The use of (TD)-DFT with the density functional BHandH to study these systems is justified for different reasons. Firstly, more extensive work done on evaluating the performance of DFT functionals for CT states showed good behaviour for the functional of our choice [18]. Secondly, we performed a comparison study between the TD-DFT calculations (BHandH/6-31G**) with DALTON and second-order approximate coupled-cluster singles and doubles (CC2) calculations $\left(6-31 \mathrm{G}^{* *}\right)$ with TURBOMOLE [19-23] on molecules 2 and 4 to verify computed trends. The CC2 calculations 
resulted in an energy difference of $0.36 \mathrm{eV}$ between $2\left(\mathrm{~S}_{1}=2.47 \mathrm{eV}\right)$ and $4\left(\mathrm{~S}_{1}=2.83 \mathrm{eV}\right)$, which is reasonably described by TD-DFT that computed an energy difference of $0.20 \mathrm{eV}$ between $2\left(\mathrm{~S}_{1}=2.41\right.$ $\mathrm{eV})$ and $4\left(\mathrm{~S}_{1}=2.61 \mathrm{eV}\right)$. Thirdly, Peach et al. [24] justified the use of a quantity called $\Lambda$ that measures the degree of spatial overlap between occupied and virtual orbitals involved in particular excitations, as a diagnostic test for judging the reliability of a general (CT) excitation energy from a particular density functional. For the hybrid functional B3LYP, the minimum value of this quantity for giving reliable results was significantly lower than the calculated values for these systems.

The vertical exciton binding energy $\mathrm{E}_{\mathrm{b}}{ }^{\mathrm{exc}}$ was defined as the energy difference between the CS state on two donor molecules (one cationic and one anionic) and the first excited state on one donor molecule using the following equation:

$\mathrm{E}_{\mathrm{b}}{ }^{\mathrm{exc}}=\mathrm{VIP}-\mathrm{VEA}-\mathrm{S}_{1}$

with VIP the vertical IP of one donor molecule, VEA the vertical EA of one donor molecule, and $\mathrm{S}_{1}$ the vertical excited state energy of one donor molecule. After the Frank-Condon photo-excitation, typical photo-physical processes like charge separation take place within only a few hundred fs [25, 26]. In order to focus solely on the effect of push-pull group substitution patterns on the lowering of the exciton binding energy (and not having the effect of geometry relaxation in addition), we calculated the vertical exciton binding energy.

Dipole moments of the vertical first excited state $S_{1}$ were calculated using Density Functional Quadratic Response Theory [27-29] (BHandH/6-31G**) with DALTON. The absolute difference in dipole moment between the ground and vertical excited state of the donor molecule, $\Delta \mu_{\mathrm{eg}}$, was calculated in the same way as introduced by Carsten et al. [8] using the following equation:

$\Delta \mu_{\mathrm{ge}}=\left[\left(\mu_{\mathrm{gx}}-\mu_{\mathrm{ex}}\right)^{2}+\left(\mu_{\mathrm{gy}}-\mu_{\mathrm{ey}}\right)^{2}+\left(\mu_{\mathrm{gz}}-\mu_{\mathrm{ez}}\right)^{2}\right]^{1 / 2}$

with e.g., $\mu_{\mathrm{gx}}$ the $\mathrm{x}$-component of the ground state dipole moment and $\mu_{\mathrm{ex}}$ the $\mathrm{x}$-component of the vertical excited state dipole moment.

In the simple Hartree-Fock (HF) picture, neglecting orbital relaxation and electron correlation effects, the CS state on two donor molecules is defined as the orbital energy difference between the LUMO and HOMO (Koopmans' theorem). In this frozen orbital (FO) model, the excitation energy for a single electron HOMO $\rightarrow$ LUMO excitation is:

$\mathrm{S}_{1}{ }^{\mathrm{HF}-\mathrm{FO}}=\Delta \varepsilon_{\mathrm{HL}}-\mathrm{J}_{\mathrm{HL}}+2 \mathrm{~K}_{\mathrm{HL}}$

with $\Delta \varepsilon_{\mathrm{HL}}$ the orbital energy difference between the HOMO and LUMO, $\mathrm{J}_{\mathrm{HL}}$ the Coulomb and $\mathrm{K}_{\mathrm{HL}}$ the exchange two-electron integrals [(HH|LL) and (HL|HL)] [30, 31]. In this HF-FO model, the exciton binding energy is thus defined as $\mathrm{J}_{\mathrm{HL}}-2 \mathrm{~K}_{\mathrm{HL}}$. Although in DFT the above analysis is not completely valid, the electron-hole interaction given by $\mathrm{J}_{\mathrm{HL}}-2 \mathrm{~K}_{\mathrm{HL}}$ can still be used as a measure of the exciton binding energy. The advantage of using $\mathrm{J}_{\mathrm{HL}}$ as this indicator is that its magnitude can be related to the spatial extent of the orbitals involved, which in turn can be related to chemical composition (a similar rationalization was applied by Van Walree et al. in [32]). Baerends et al. [33] state that the shape of the KS orbitals describes excited states very well, justifying the use of KS orbitals in this respect.

To get insight in the degree of electron-hole separation and to judge the reliability of the excitation energies from the density functional $\mathrm{BHandH}$, the degree of spatial overlap $\Lambda$ between occupied and virtual orbitals involved in the excitation was determined. The quantity $\Lambda$ as defined in DALTON was used: 
with $\kappa_{\mathrm{ia}}$ the contribution of an occupied-virtual pair to a given TD-DFT excitation and $\mathrm{O}_{\mathrm{ia}}$ the inner product of the moduli of a given occupied orbital $\varphi_{\mathrm{i}}$ and a virtual orbital $\varphi_{\mathrm{a}}$, which is weighted by $\kappa^{2}{ }_{\mathrm{ia}}$ [24]. Small values of $\Lambda$ correspond to long-range excitations and large values to short-range excitations. When $\Lambda$ falls below a prescribed threshold, for example $\Lambda<0.3$ for B3LYP, excitation energies are likely to be in very significant error. It should be noted that the quantity $\Lambda$ is not unique, so this diagnostic number is qualitative [24].

Since all calculations were done on isolated monomers, the theoretical values for all molecular energies are higher compared to experimental values because stabilisation effects from the environment are missing. However, since we are looking at differences in excited state properties between the monomers rather than absolute values, resulting trends still hold, also when environmental effects are incorporated.

\section{Results and discussion}

\subsection{Monomers}

\subsubsection{Geometry of molecules 1-6}

Geometry optimization of molecules 1-6 resulted in slight deviations from planarity for the substituted molecules 2-6. Two dihedral angles (Fig. 4 and Table 1) were defined to investigate if conjugation still applies to the molecules. Dihedral angle $\alpha$ is similar for all substituted molecules 2-6 and dihedral angle $\beta$ clearly is larger for the linear-conjugated molecules (LCM) $\mathbf{2 - 3}$ compared to the cross-conjugated molecules (CCM) 4-5. This difference is caused by the position of $\mathrm{NO}_{2}$ in 2-3, i.e., it is attached closer to the thiophene spacer, which causes more steric hindrance. Despite this moderate loss of planarity, the interaction between the p-orbitals that form the $\pi$-bonds is still significant (it scales with cos[angle]), so the push-pull groups are conjugated.

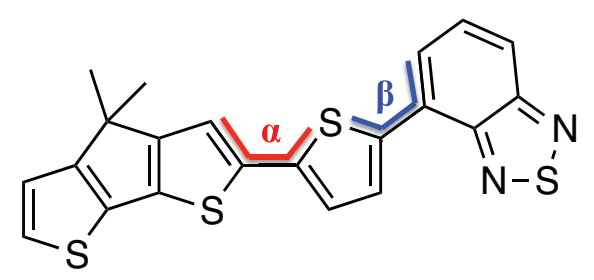

Fig. 4. Molecule 1 with defined dihedral angles $\alpha$ (red) and $\beta$ (blue).

Table 1. Dihedral angle $\alpha\left({ }^{\circ}\right.$, Fig. 4$)$ and dihedral angle $\beta\left({ }^{\circ}\right.$, Fig. 4$)$ for molecules 1-6.

\begin{tabular}{ccc}
\hline Molecule & Dihedral angle $\boldsymbol{\alpha}$ & Dihedral angle $\boldsymbol{\beta}$ \\
\hline $\mathbf{1}$ & 0.0 & 0.0 \\
$\mathbf{2}$ & -24.2 & 34.2 \\
$\mathbf{3}$ & -22.2 & 37.9 \\
$\mathbf{4}$ & -13.1 & 2.1 \\
$\mathbf{5}$ & -20.5 & 4.0 \\
$\mathbf{6}$ & -25.9 & -5.4 \\
\hline
\end{tabular}




\subsubsection{Molecular orbitals of molecules 1-6}

The HOMO and LUMO of molecules 1-6 (Fig. 5) reveal an interesting trend: for the LCM 2-3 the HOMOs and LUMOs are both delocalised over the carbon atoms to which the $\mathrm{NH}_{2}$ and $\mathrm{NO}_{2}$ groups are attached. This is contrary to the CCM 4-5 where the HOMOs are delocalised over carbon atoms to which the $\mathrm{NH}_{2}$ group is attached and the LUMOs over carbon atoms to which the $\mathrm{NO}_{2}$ group is attached. The only exception is the LUMO of LCM 2, which is not delocalised over the carbon atom to which the $\mathrm{NH}_{2}$ group is attached.

For the LCM 2-3, the HOMOs are delocalised over the donating fragment CPDT (and $\mathrm{NH}_{2}$ ) and the central thiophene spacer T, but also over two carbon atoms of the accepting fragment BT. One of these is the one to which the $\mathrm{NO}_{2}$ group is attached. The LUMO of $\mathbf{3}$ is delocalised over the accepting fragment $\mathrm{BT}$ (and $\mathrm{NO}_{2}$ ) and also over two carbon atoms of the central thiophene spacer T. Amongst these is the one to which the $\mathrm{NH}_{2}$ group is attached. The LUMO of $\mathbf{2}$ is also delocalised over the BT unit (and $\mathrm{NO}_{2}$ ) and partly over the central thiophene spacer $\mathrm{T}$, but for $\mathbf{2}$ the $\mathrm{NH}_{2}$ group is not connected to this spacer. For the CCM 4-5, the HOMOs are also delocalised over the donating fragment CPDT (and $\mathrm{NH}_{2}$ ), the central thiophene spacer $\mathrm{T}$ and over two carbon atoms of the accepting fragment BT. However, for 4-5 these carbon atoms are not those to which the $\mathrm{NO}_{2}$ group is attached. The LUMOs of 4-5 are also delocalised over the accepting fragment $\mathrm{BT}$ (and $\mathrm{NO}_{2}$ ) and over two carbon atoms of the central thiophene spacer $\mathrm{T}$, but again these carbon atoms are not attached to the $\mathrm{NH}_{2}$ group.

This trend can be understood from simple Hückel theory [34] applied to the simplest, odd alternating hydrocarbon system that models the thiophene-benzene part of the monomers, namely methylenebenzene (Fig. 6). Its non-bonding molecular orbital (NBMO) can be determined without solving the secular equations. This HOMO has small electron density at the meta positions and large electron density at the ortho/para positions, resulting in an ortho/para directing, activating towards electrophilic substitution (e.g., of $\mathrm{NO}_{2}$ ) effect of - in this case - the by resonance electron donating methylene substituent. This result of the Hückel analysis is irrespective of the size of the ring. It demonstrates clearly that linear-conjugation corresponding to ortho/para substitution leads to more $\pi$ delocalised HOMO and LUMO over similar parts of the molecule. In contrary, cross-conjugation corresponding to meta substitution leads to less delocalised frontier MOs over separate parts of the molecule.

Molecule $\mathbf{6}$ shows clearly that functional groups that exert inductive effects do not participate in the frontier MOs, because these orbitals are $\pi$-type. Consequently the frontier MOs are very similar to the ones of molecule 1. 


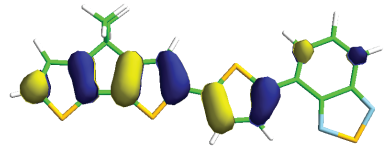

HOMO 1

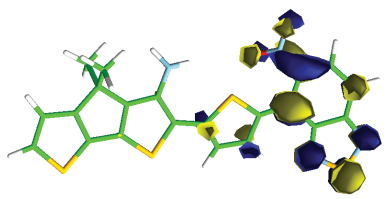

LUMO 2

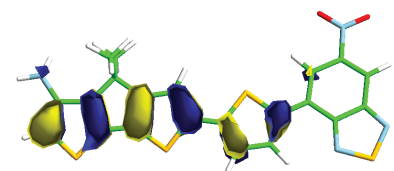

HOMO 4

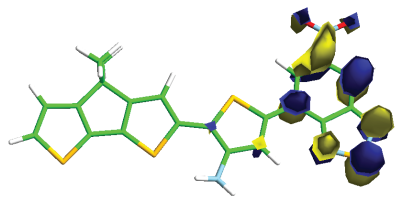

LUMO 5

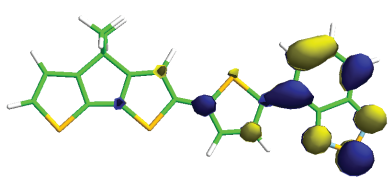

LUMO 1

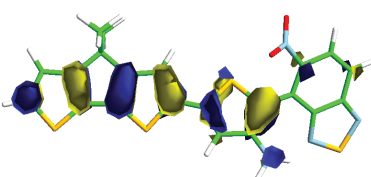

HOMO 3

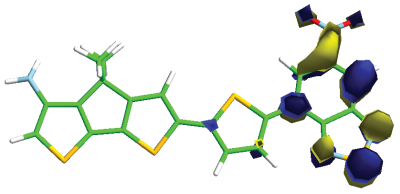

LUMO 4

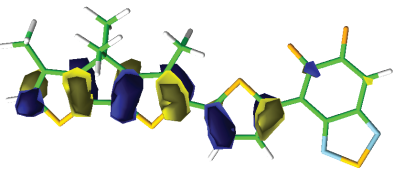

HOMO 6

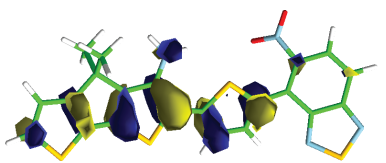

HOMO 2

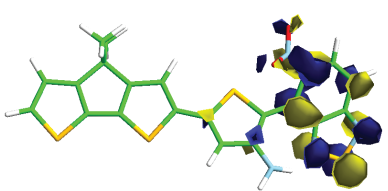

LUMO 3

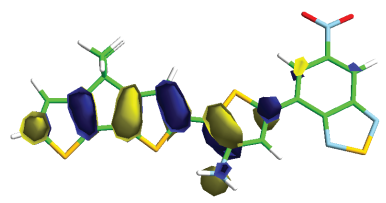

HOMO 5

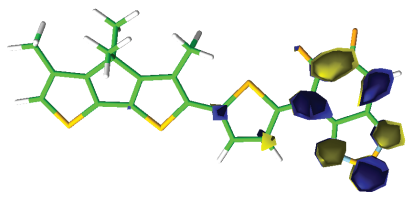

LUMO 6

Fig. 5. Frontier molecular orbitals of molecules 1-6. For all molecules, the first excited state corresponds predominantly to a HOMO $\rightarrow$ LUMO transition $\left(\mathrm{c}^{2}>0.83\right)$.<smiles>CC1CCCCC1C</smiles>

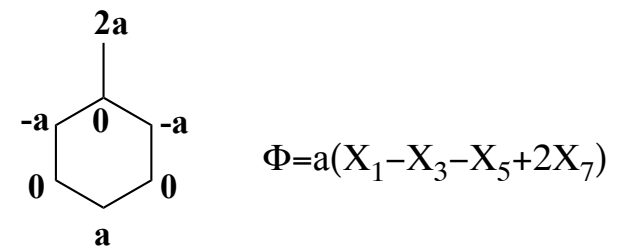

NBMO coefficients

Fig. 6. Example of an odd alternating hydrocarbon, methylenebenzene (left), and its non-bonding molecular orbital $\Phi$ (right) within the Hückel approximation. 


\subsubsection{Excited state properties of molecules 1-6}

A summary of the excited state properties of interest for molecules 1-6 is given in Table 2. The vertical excitation energies of the substituted molecules 2-6 for the HOMO $\rightarrow$ LUMO transition decrease with respect to the reference molecule 1. Not surprisingly, a larger effect is found if the substituents interfere with the $\pi$-system of the pristine molecule (2-5), namely a decrease in the range of $0.3-0.5 \mathrm{eV}$. Only a slight decrease of $0.03 \mathrm{eV}$ is found for molecule 6, which has functional groups that exert inductive effects. For all molecules 2-6, the oscillator strength decreases compared to reference molecule 1. A large decrease is observed for 2-5 and a small decrease for $\mathbf{6}$. No clear distinction between linear- and cross-conjugation is discernible.

For all monomers, the bandgap $\Delta \varepsilon_{\mathrm{HL}}$ becomes smaller, mostly because of lowering of the $\varepsilon_{\mathrm{LUMO}}$. This lowering is the smallest for $\mathbf{6}$. No correlation is found between $S_{1}$ and $\Delta \varepsilon_{\mathrm{HL}}$, which can be explained by realising that not only $\Delta \varepsilon_{\mathrm{HL}}$ contributes to $\mathrm{S}_{1}$ but also the electron-hole interaction (Eq. (3)). We come back to this point later when the results of the Coulomb and exchange energies are discussed.

A good correspondence in the trends for 1-6 between VIP and $\varepsilon_{\text {Номо }}$ and between VEA and $\varepsilon_{\text {LUMO }}$ is found, as expected. When the results of the LCM 2-3 are compared to the ones of the CCM 4-5, only a difference in $\varepsilon_{\mathrm{LUMO}}$ (and VEA) is found: the $\varepsilon_{\mathrm{LUMO}}$ is higher (and the VEA is smaller) for the LCM 2-3. This difference could be explained as follows. As noticed earlier, for the LCM 2-3 both the HOMOs and the LUMO of 3 are delocalised over the carbon atoms to which the $\mathrm{NH}_{2}$ and $\mathrm{NO}_{2}$ groups are attached. So for their $\varepsilon_{\mathrm{LUMO}}$ the stabilizing effect of $\mathrm{NO}_{2}$ (Fig. 7) is opposed by the destabilizing effect of $\mathrm{NH}_{2}$ (Fig. 7), leading to relatively high $\varepsilon_{\text {LUMO }}$ of the LCM 2-3 compared to the CCM 4-5. Vice versa, for the $\varepsilon_{\text {номо }}$ of the LCM 2-3 the destabilizing effect of the $\mathrm{NH}_{2}$ is opposed by the stabilizing effect of the $\mathrm{NO}_{2}$, leading to relatively low $\varepsilon_{\text {номо }}$ compared to the CCM 4-5. CCM 4 does

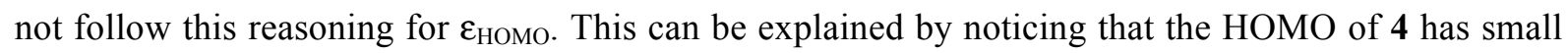
electron density at the carbon atom to which $\mathrm{NH}_{2}$ is attached and is less delocalised over $\mathrm{NH}_{2}$ compared to the HOMO of the other CCM 5 (Fig. 5). Consequently the destabilizing effect of $\mathrm{NH}_{2}$ for the HOMO is very small in $\mathbf{4}$, leading to a relatively low $\varepsilon_{\mathrm{HOMO}}$ compared to $\mathbf{5}$.

The inductive effects of $\mathrm{CH}_{3}$ and $\mathrm{F}$ of $\mathbf{6}$ have no significant effect on the vertical excitation energies, HOMO/LUMO energies, VIP and VEA, compared to 1.

Table 2. The excitation energy $\left(\mathrm{S}_{1}, \mathrm{eV}\right)$ to the first excited state ${ }^{*}$, the oscillator strength $(f)$, the HOMO energy $\left(\varepsilon_{\text {HOMO }}, \mathrm{eV}\right)$, the LUMO energy $\left(\varepsilon_{\mathrm{LUMO}}, \mathrm{eV}\right)$, the orbital energy difference between the HOMO and LUMO $\left(\Delta \varepsilon_{\mathrm{HL}}, \mathrm{eV}\right)$, the vertical ionization potential (VIP, eV), and the vertical electron affinity (VEA, eV) for molecules 1-6.

\begin{tabular}{|c|c|c|c|c|c|c|c|}
\hline Molecule & $\mathbf{S}_{1}$ & $f$ & $\varepsilon_{\text {номо }}$ & $\varepsilon_{\text {LUMO }}$ & $\Delta \varepsilon_{\mathrm{HL}}$ & VIP & VEA \\
\hline 1 & 2.90 & 0.803 & -5.72 & -1.44 & 4.28 & 6.33 & 0.72 \\
\hline \multicolumn{8}{|c|}{ linear-conjugation, mesomeric effect } \\
\hline 2 & 2.41 & 0.581 & -5.74 & -1.98 & 3.75 & 6.30 & 1.29 \\
\hline 3 & 2.36 & 0.373 & -5.85 & -2.00 & 3.85 & 6.44 & 1.28 \\
\hline \multicolumn{8}{|c|}{ cross-conjugation, mesomeric effect } \\
\hline 4 & 2.61 & 0.520 & -5.86 & -2.13 & 3.73 & 6.46 & 1.40 \\
\hline 5 & 2.37 & 0.473 & -5.64 & -2.16 & 3.48 & 6.23 & 1.44 \\
\hline \multicolumn{8}{|c|}{ inductive effect } \\
\hline 6 & 2.87 & 0.725 & -5.69 & -1.53 & 4.16 & 6.36 & 0.76 \\
\hline
\end{tabular}

*these excitations all correspond to a HOMO $\rightarrow$ LUMO transition $\left(c^{2}>0.83\right)$ 

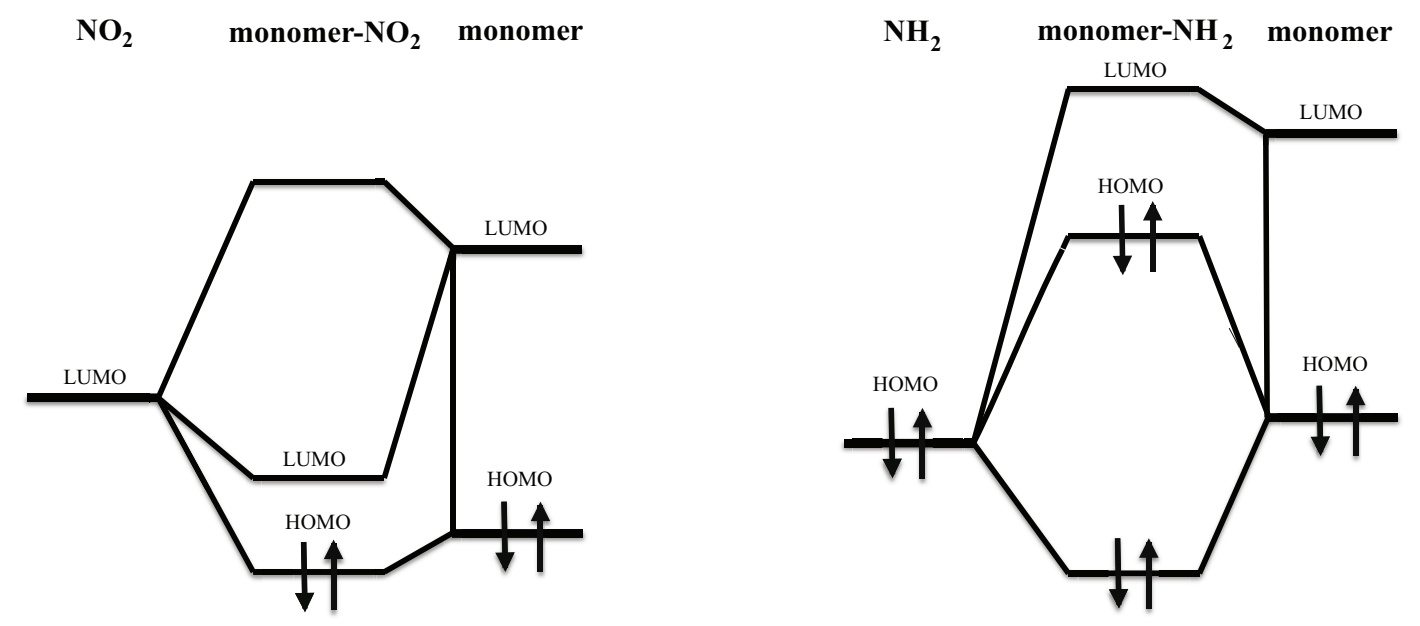

Fig. 7. Molecular orbital energy levels diagrams showing the stabilising effect of the LUMO of $\mathrm{NO}_{2}$ (left) and the destabilising effect of the HOMO of $\mathrm{NH}_{2}$ (right) on the HOMO and LUMO of the monomer.

\subsubsection{Properties relevant for OPV applications of molecules 1-6}

A summary of the properties relevant for OPV applications of molecules 1-6 is given in Table 3. The electron-hole interaction calculated as $\left[\mathrm{J}_{\mathrm{HL}}-2 \mathrm{~K}_{\mathrm{HL}}\right]$ decreases by $0.5 \mathrm{eV}$ for the CCM 4-5 and increases by $0.1 \mathrm{eV}$ for the LCM 2-3, compared to the reference molecule $\mathbf{1}$. Only a slight decrease is found for the $\mathrm{CH}_{3} / \mathrm{F}$ substituted molecule 6. This significant difference in $\left[\mathrm{J}_{\mathrm{HL}}-2 \mathrm{~K}_{\mathrm{HL}}\right]$ of the CCM 4-5 compared to the LCM 2-3 can be explained by looking at the overlap density between the orbitals involved in the first excited state (Fig. 5). The overlap density between the HOMO and LUMO is smaller for the CCM 4-5 than for the LCM 2-3 leading to smaller Coulomb and exchange terms. This trend in overlap density is confirmed by the trend in the quantity $\Lambda$, which is significantly smaller for the CCM 4-5 compared to the LCM 2-3. This rationalization shows that $\left[\mathrm{J}_{\mathrm{HL}}-2 \mathrm{~K}_{\mathrm{HL}}\right]$ can indeed be related to the spatial extent of the orbitals involved in the excitation and thus to chemical composition. It is also in perfect correspondence with the earlier explained analysis of linear-conjugation (and crossconjugation) using Hückel theory, leading to a more (and less) $\pi$-delocalised HOMO and LUMO over similar (and different) parts of the molecule. In general it is known that cross-conjugation does not result in pronounced $\pi$-electron delocalisation, leading to a relatively large reduction in electron repulsion upon excitation [35].

The vertical exciton binding energy decreases for all molecules in the range $0.1-0.3 \mathrm{eV}$ except for 3 and 6, compared to the reference molecule 1. The decrease is the largest for the CCM 4-5. For the LCM 3 the vertical exciton binding energy increases by $0.1 \mathrm{eV}$ and for molecule $\mathbf{6}$ it does not change significantly. When these values are compared to the ones of $\left[\mathrm{J}_{\mathrm{HL}}-2 \mathrm{~K}_{\mathrm{HL}}\right]$, a reasonably similar trend is found: from linear- to cross-conjugation, the $\left[\mathrm{J}_{\mathrm{HL}}-2 \mathrm{~K}_{\mathrm{HL}}\right]$ decreases by $0.4-0.5 \mathrm{eV}$, which is similar to the decrease in the vertical exciton binding energy.

Concerning the last interesting property relevant for OPV applications, the change in dipole moment upon excitation increases for all molecules except for 3, compared to the reference molecule 1. This increase is the largest for the CCM 4-5 and the smallest for the LCM 2. 
Table 3. The electron-hole interaction calculated as $\left[\mathrm{J}_{\mathrm{HL}}-2 \mathrm{~K}_{\mathrm{HL}}\right](\mathrm{eV})$, the degree of spatial overlap between occupied and virtual orbitals involved in the excitation ( $\Lambda$, Eq. (4)), the vertical exciton binding energy $\left(\mathrm{E}_{\mathrm{b}}{ }^{\mathrm{exc}}\right.$, Eq. (1), eV), and the change in dipole moment upon excitation $\left(\Delta \mu_{\mathrm{ge}}\right.$, Eq. (2), Debye) for molecules 1-6.

\begin{tabular}{ccccc}
\hline Molecule & $\mathbf{J}_{\mathbf{H L}} \mathbf{- 2} \mathbf{K}_{\mathbf{H L}}$ & $\boldsymbol{\Lambda}$ & $\mathbf{E}_{\mathbf{b}}{ }^{\mathbf{e x c}}$ & $\boldsymbol{\Delta} \boldsymbol{\mu}_{\mathbf{g e}}$ \\
\hline $\mathbf{1}$ & 2.47 & 0.57 & 2.70 & 11.11 \\
$\mathbf{2}$ & 2.52 & 0.48 & 2.61 & 11.53 \\
$\mathbf{3}$ & 2.58 & 0.46 & 2.79 & 10.13 \\
$\mathbf{4}$ & 1.98 & 0.39 & 2.46 & 17.84 \\
$\mathbf{5}$ & 2.06 & 0.38 & 2.42 & 17.57 \\
$\mathbf{6}$ & 2.34 & 0.51 & 2.73 & 13.47 \\
\hline
\end{tabular}

Let us now study possible relations between properties related to chemical composition and observables that are important for specific photo-physical applications. Part of this work focuses on OPV applications and in this context the exciton binding energy is an important property. Firstly, the statement made in the introduction about the relation between a more separated exciton and a larger dipole moment change upon excitation is investigated [8-10]. Although in DFT the electron-hole interaction given by $\left[\mathrm{J}_{\mathrm{HL}}-2 \mathrm{~K}_{\mathrm{HL}}\right]$ can only be used as a measure of the exciton binding energy, a perfect linear correlation between $\left[\mathrm{J}_{\mathrm{HL}}-2 \mathrm{~K}_{\mathrm{HL}}\right]$ and $\Delta \mu_{\mathrm{ge}}$ is found (Fig. 8). As showed earlier, both are related to spatial extent of the orbitals involved in the excitation and thus to chemical composition.

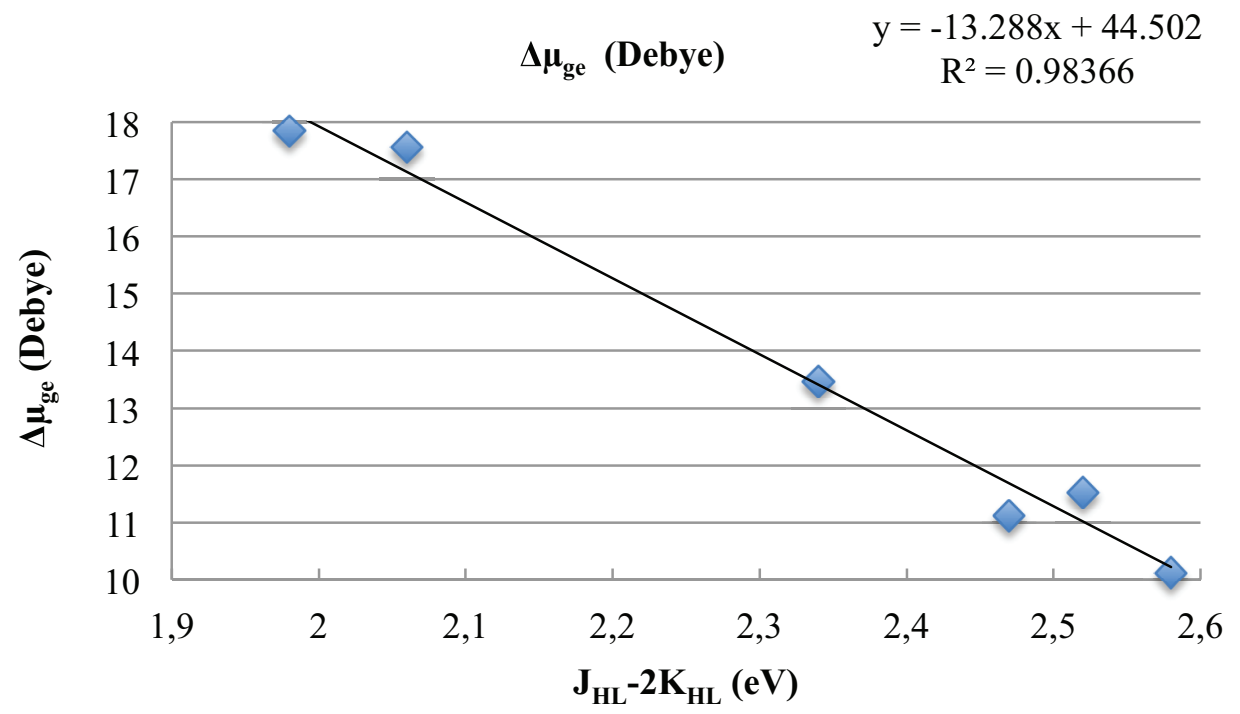

Fig. 8. Change in dipole moment upon excitation ( $\Delta \mu_{\mathrm{ge}}$, Debye) as a function of the electron-hole interaction $\left(\left[\mathrm{J}_{\mathrm{HL}}-2 \mathrm{~K}_{\mathrm{HL}}\right], \mathrm{eV}\right)$. A linear trend line is added together with $\mathrm{R}^{2}$.

As is also stated in the introduction, a direct relation has been found by Carsten et al. [8] between the device efficiency and $\Delta \mu_{\mathrm{ge}}$, if a larger $\Delta \mu_{\mathrm{ge}}$ is the result of a more separated exciton (i.e., an exciton with a smaller binding energy). Secondly, the relation between these two properties is studied. Although $\mathrm{R}^{2}$ is 0.80 , still a linear correlation is found (Fig. 9), but not as nicely as the one found between $\left[\mathrm{J}_{\mathrm{HL}}-2 \mathrm{~K}_{\mathrm{HL}}\right]$ and $\Delta \mu_{\mathrm{ge}}$ (Fig. 8). These findings suggest that important photo-physical properties, like the exciton binding energy, can be engineered by tuning of properties related to chemical composition (e.g., $\left[\mathrm{J}_{\mathrm{HL}}-2 \mathrm{~K}_{\mathrm{HL}}\right]$ and $\Delta \mu_{\mathrm{ge}}$ ). 


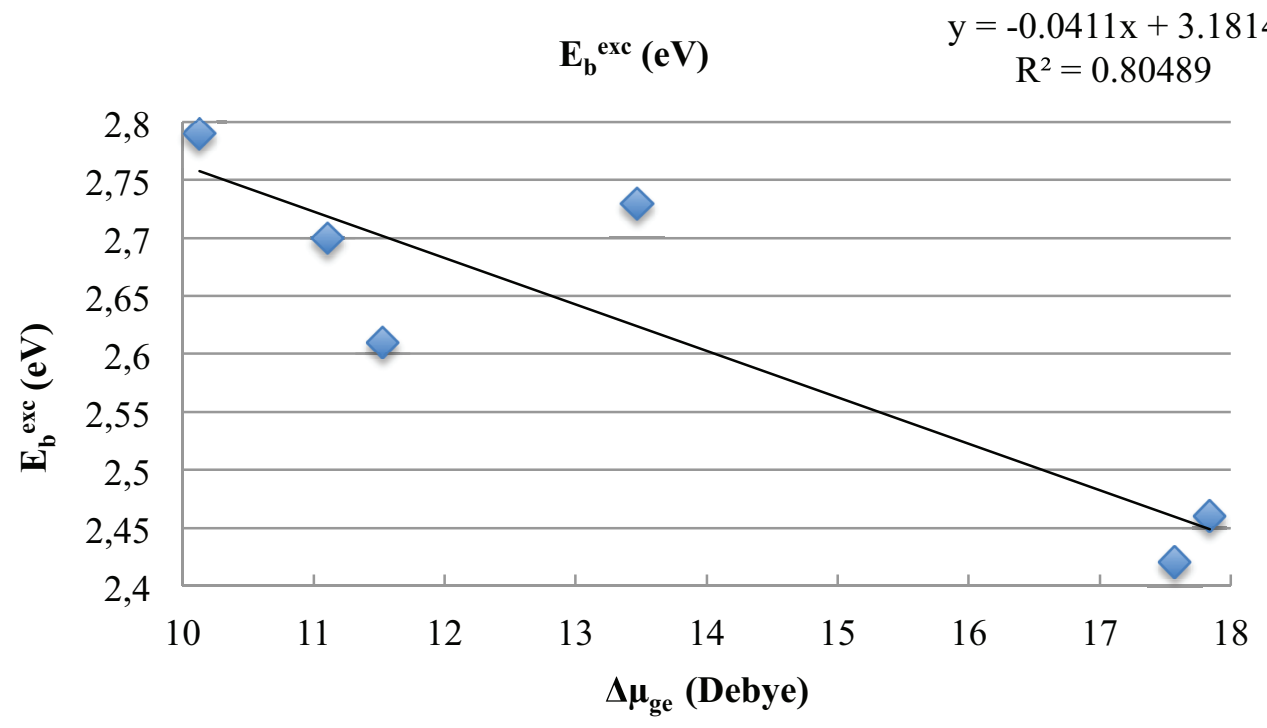

Fig. 9. The vertical exciton binding energy $\left(\mathrm{E}_{\mathrm{b}}{ }^{\mathrm{exc}}, \mathrm{eV}\right)$ as a function of the change in dipole moment upon excitation $\left(\Delta \mu_{\text {ge }}\right.$, Debye). A linear trend line is added together with $\mathrm{R}^{2}$.

\subsection{Trimers}

\subsubsection{Geometry of molecules 7-12}

Geometry optimization of trimers 7-12 resulted in different values for dihedral angle $\gamma$ (Fig. 10 and Table 4) between two subsequent monomers. The loss of planarity is the largest for 10, 11 and $\mathbf{1 2}$, probably caused by the position of $\mathrm{NO}_{2} / \mathrm{F}$ with respect to the CPDT unit. Despite this loss of planarity for all trimers 7-12, the interaction between neighbouring monomers is still sufficient to speak of conjugation.

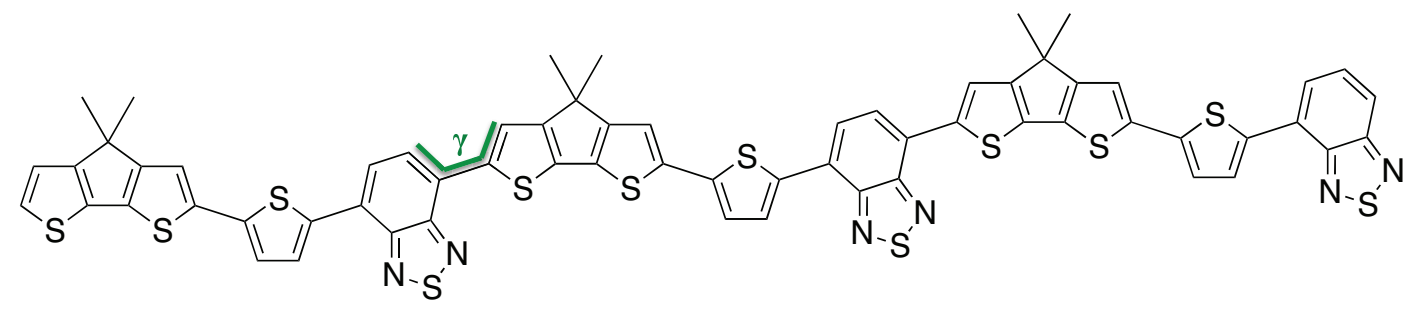

Fig. 10. Molecule 7 with defined dihedral angle $\gamma$ (green).

Table 4. Dihedral angle $\gamma\left({ }^{\circ}\right.$, Fig. 10) for molecules 7-12.

\begin{tabular}{cc}
\hline Molecule & Dihedral angle $\boldsymbol{\gamma}$ \\
\hline $\mathbf{7}$ & -4.0 \\
$\mathbf{8}$ & -2.7 \\
$\mathbf{9}$ & -5.3 \\
$\mathbf{1 0}$ & -31.0 \\
$\mathbf{1 1}$ & -23.4 \\
$\mathbf{1 2}$ & -38.2 \\
\hline
\end{tabular}




\subsubsection{Molecular orbitals of molecules 7-12}

For trimers 7-12 all occupied-virtual orbital pairs that correspond to the first excited state with $\mathrm{c}^{2}>$ 0.20 were analysed (Fig. S1 in Supplementary material). For trimers 7, 8, 9 and 12 the first excited state corresponds to a HOMO $\rightarrow$ LUMO transition $\left(c^{2}>0.62\right)$. For trimer 10, the HOMO- $\rightarrow$ LUMO transition becomes equally important and for trimer $\mathbf{1 1}$ the HOMO $\rightarrow$ LUMO+1 transition becomes considerably important. When these orbitals are compared to the ones of the monomers, it is noticed that both HOMO and LUMO are more delocalised. The HOMOs of the trimers cannot be regarded as simple superpositions of the HOMOs of their corresponding monomers. In general the electron density of the HOMOs of the trimers is the largest on the donating fragment of the second monomer. The reference trimer 7 has the most delocalised HOMO with comparable electron density on the second and third donating fragment. Trimers 8-9 have HOMOs with also considerable electron density on the first donating fragment. The LUMOs of 7-12 can be more or less regarded as superpositions of the LUMOs of 1-6 because they are delocalised over all three accepting fragments, especially for trimer $\mathbf{9}$. For the other trimers, the LUMOs have the largest electron density on the accepting fragment of the second monomer.

For the trimers, the computed difference between linear- and cross-conjugation concerning HOMO/LUMO delocalisation over both or either $\mathrm{NH}_{2} / \mathrm{NO}_{2}$ is not retrieved, because the distinction in conjugation pattern does not hold anymore. The only difference between trimers 8-9 (derived from the LCM 2-3) and trimers 10-11 (derived from the CCM 4-5) is that the HOMOs of trimers 10-11 are less delocalised than the ones of 7-9 and $\mathbf{1 2}$ (i.e., 8-9: HOMO is delocalised over the first and second donating fragment; 10-11: HOMO is localised predominantly on the second donating fragment; 7-12: HOMO is delocalised on the second and third donating fragment). It is also noticed that for trimers 1011 the HOMOs and LUMOs are more localised at different parts of the molecules, leading to relatively smaller overlap densities between these orbitals, compared to trimers 8-9.

\subsubsection{Excited state properties of molecules 7-12}

A summary of the excited state properties of interest for trimers 7-12 is given in Table 5 . When the values of Table 5 are compared to the ones for the monomers (Table 1), it is noticed that in general the vertical excitation energies are lowered by about $0.4-0.8 \mathrm{eV}$ and the relative differences between molecules are diminished. Compared to the monomers, the oscillator strengths increase by a factor 45. All bandgaps of the trimers are smaller by about $0.5-0.6 \mathrm{eV}$ (except for 7: $0.9 \mathrm{eV}$ ) compared to the monomers, because their $\varepsilon_{\text {НОмо }}$ increase by about $0.3-0.4 \mathrm{eV}$ and their $\varepsilon_{\mathrm{LUMO}}$ decrease by about $0.2-0.4$ $\mathrm{eV}$. Consequently the VIPs of the trimers decrease by $0.6-0.8 \mathrm{eV}$ and their VEAs increase by $0.6-0.8$ $\mathrm{eV}$. These results are explained by the fact that in general chain extension destabilises $\varepsilon_{\text {Hомо }}$ and stabilises $\varepsilon_{\text {LUMO. }}$

The following trends are found for the trimers. The vertical excitation energies for the HOMO $\rightarrow$ LUMO transition of the $\mathrm{NH}_{2} / \mathrm{NO}_{2}$ substituted trimers 8-11 decrease slightly by about $0.1-0.2 \mathrm{eV}$ compared to the reference trimer 7. For trimer 12, with $\mathrm{CH}_{3} / \mathrm{F}$ functional groups, an increase of $0.2 \mathrm{eV}$ is found. For all $\mathrm{NH}_{2} / \mathrm{NO}_{2}$ substituted trimers 8-11 the oscillator strength decreases compared to the reference 7 and for the $\mathrm{CH}_{3} / \mathrm{F}$ substituted molecule 12 the oscillator strength increases slightly. $\mathrm{NH}_{2} / \mathrm{NO}_{2}$ substituted trimers 8-11 have a smaller bandgap and the $\mathrm{CH}_{3} / \mathrm{F}$ substituted trimer $\mathbf{1 2}$ has a larger bandgap, compared to the reference trimer 7. This trend is in accordance to the one for the vertical excitation energy. A perfect correspondence in trends for 7-12 between VIP and $\varepsilon_{\text {HOMO }}$ and between VEA and $\varepsilon_{\text {LUMO }}$ is found. It is noticed that both the VIP and VEA increase (except for trimer 12: the VEA decreases), compared to the reference 7. 
When these trends are compared to the ones for the monomers, some differences are found. For trimer 12, the vertical excitation energy increases compared to 7, whereas for monomer 6 it does not change compared to $\mathbf{1}$. Also for the oscillator strength trimer $\mathbf{1 2}$ shows different behaviour compared to monomer 6: the trimer oscillator strength increases, whereas the monomer oscillator strength decreases slightly. In contrast to the monomers, the trend for the vertical excitation energy corresponds rather well to the one for the bandgap $\Delta \varepsilon_{\mathrm{HL}}$. Finally, also in contrast to the monomers, for trimer 12 the inductive effects of the $\mathrm{CH}_{3} / \mathrm{F}$ groups appear to have a significant effect on the vertical excitation energy, the HOMO/LUMO energies, VIP and VEA.

Table 5. The excitation energy $\left(\mathrm{S}_{1}, \mathrm{eV}\right)$ to the first excited state ${ }^{*}$, the oscillator strength $(f)$, the HOMO energy $\left(\varepsilon_{\text {HOMO }}, \mathrm{eV}\right)$, the LUMO energy $\left(\varepsilon_{\mathrm{LUMO}}, \mathrm{eV}\right)$, the orbital energy difference between the HOMO and LUMO $\left(\Delta \varepsilon_{\mathrm{HL}}, \mathrm{eV}\right)$, the vertical ionization potential (VIP, eV), and the vertical electron affinity (VEA, eV) for molecules 7-12.

\begin{tabular}{|c|c|c|c|c|c|c|c|}
\hline Molecule & $\mathbf{S}_{1}$ & $f$ & $\varepsilon_{\text {номо }}$ & $\varepsilon_{\text {LUMO }}$ & $\Delta \varepsilon_{\mathrm{HL}}$ & VIP & VEA \\
\hline 7 & 2.12 & 3.780 & -5.29 & -1.87 & 3.42 & 5.58 & 1.54 \\
\hline \multicolumn{8}{|c|}{ mesomeric effect } \\
\hline 8 & 1.91 & 3.099 & -5.44 & -2.29 & 3.15 & 5.70 & 1.98 \\
\hline 9 & 1.90 & 2.326 & -5.50 & -2.29 & 3.21 & 5.78 & 1.96 \\
\hline 10 & 2.01 & 3.002 & -5.53 & -2.32 & 3.21 & 5.84 & 1.98 \\
\hline 11 & 1.85 & 3.203 & -5.33 & -2.37 & 2.97 & 5.61 & 2.04 \\
\hline \multicolumn{8}{|c|}{ inductive effect } \\
\hline 12 & 2.34 & 3.862 & -5.42 & -1.77 & 3.66 & 5.72 & 1.43 \\
\hline
\end{tabular}

these excitations all correspond to a HOMO $\rightarrow$ LUMO transition with $\mathrm{c}^{2}>0.62$, except for $\mathbf{1 0}$ $\left(\right.$ HOMO $\rightarrow$ LUMO with $\mathrm{c}^{2}=0.25$ and HOMO- $\rightarrow$ LUMO with $\left.\mathrm{c}^{2}=0.26\right)$ and $\mathbf{1 1}(\mathrm{HOMO} \rightarrow$ LUMO with $\mathrm{c}^{2}=0.37$ and HOMO $\rightarrow$ LUMO +1 with $\mathrm{c}^{2}=0.22$ )

\subsubsection{Properties relevant for OPV applications of molecules 7-12}

A summary of the properties relevant for OPV applications of trimers 7-12 is given in Table 6. The same properties as the ones for the monomers were analysed, except for the electron-hole interaction calculated as $\left[\mathrm{J}_{\mathrm{HL}}-2 \mathrm{~K}_{\mathrm{HL}}\right]$ because not for all trimers the first excited state can be regarded as predominantly a one-electron HOMO $\rightarrow$ LUMO transition.

The degree of spatial overlap between occupied and virtual orbitals involved in the excitation, $\Lambda$, is smaller for trimers 10-11 (originally derived from CCM) than for trimers 8-9 (originally derived from LCM). This difference is also found in the case of the monomers. In general the values for $\Lambda$ are larger for the trimers than for the monomers, which is explained by the larger delocalisation of the occupied and virtual orbitals involved in the excitation over more than one repeating unit.

The vertical exciton binding energies are smaller compared to values for the monomers. Because the HOMO and LUMO of trimers are more delocalised (Fig. S1 in Supplementary material), the electron-hole attraction is reduced. For the trimers, the vertical exciton binding energy decreases slightly for the $\mathrm{NH}_{2} / \mathrm{NO}_{2}$ substituted trimers 8 and 10-11, compared to reference 7. The largest decrease is found for trimer 11, namely $0.2 \mathrm{eV}$, which correlates with the results for the monomers: corresponding monomer 5 also shows the largest decrease, namely of $0.3 \mathrm{eV}$. Trimer $\mathbf{9}$ shows no significant change in vertical exciton binding energy, whereas its corresponding monomer $\mathbf{3}$ shows an increase of about $0.1 \mathrm{eV}$. This difference in behaviour between monomer and trimer is explained by realising that in the trimer the excitation is more delocalised because electron density transfers to two different parts of the molecule (Fig. S1 in Supplementary material). For the $\mathrm{CH}_{3} / \mathrm{F}$ substituted trimer 
12 the change in vertical exciton binding energy is not significant, similar to what is found for its corresponding monomer 6. In general the computed trend in vertical exciton binding energy for the monomers holds reasonably for the trimers.

Noteworthy is the relatively high vertical exciton binding energy of trimer 10, compared to the relatively low value for its corresponding monomer 4 . This result can be explained by looking at the $\mathrm{NH}_{2} / \mathrm{NO}_{2}$ group distance inside (i.e., intra) the monomer and the one between two neighbouring (i.e., inter) monomers. For monomer 4, the intramonomer push-pull distance is relatively large and as Fig. 5 shows, the HOMO $\rightarrow$ LUMO excitation takes place over a relatively large distance from the donating to the accepting fragment. For trimer 10, the intermonomer push-pull distance is very small and as Fig. S1 in Supplementary material shows, during the HOMO $\rightarrow$ LUMO excitation electron density transfers from $\mathrm{NH}_{2}$ to $\mathrm{NO}_{2}$ that are closely positioned.

The change in dipole moment upon excitation increases for all trimers, compared to the reference 7. When comparing to the monomers, again a larger increase is found for the $\mathrm{NH}_{2} / \mathrm{NO}_{2}$ substituted trimers 8-11 than for the $\mathrm{CH}_{3} / \mathrm{F}$ substituted trimer 12. The overall magnitudes of and the differences between dipole moment change upon excitation are smaller. The relatively large value for trimer 9 (compared to monomer $\mathbf{3}$ ) arises from the fact that for the trimer the excitation is more delocalised: electron density transfers to two different parts of the molecule. The relatively low value for trimer $\mathbf{1 0}$ (compared to monomer 4) is because electron density transfers from $\mathrm{NH}_{2}$ to $\mathrm{NO}_{2}$ that are closely positioned.

Table 6. The degree of spatial overlap between occupied and virtual orbitals involved in the excitation $\left(\Lambda\right.$, Eq. (4)), the vertical exciton binding energy $\left(\mathrm{E}_{\mathrm{b}}{ }^{\mathrm{exc}}, \mathrm{Eq} .(1), \mathrm{eV}\right)$ and the change in dipole moment upon excitation $\left(\Delta \mu_{\mathrm{ge}}\right.$, Eq. (2), Debye) for molecules 7-12.

\begin{tabular}{cccc}
\hline Molecule & $\boldsymbol{\Lambda}$ & $\mathbf{E}_{\mathbf{b}}{ }^{\mathbf{e x c}}$ & $\boldsymbol{\Delta} \boldsymbol{\mu}_{\mathbf{g e}}$ \\
\hline $\mathbf{7}$ & 0.66 & 1.91 & 2.14 \\
$\mathbf{8}$ & 0.56 & 1.81 & 3.77 \\
$\mathbf{9}$ & 0.54 & 1.92 & 4.14 \\
$\mathbf{1 0}$ & 0.44 & 1.85 & 2.78 \\
$\mathbf{1 1}$ & 0.44 & 1.72 & 3.79 \\
$\mathbf{1 2}$ & 0.59 & 1.95 & 2.54 \\
\hline
\end{tabular}

Finally, the relation between dipole moment change upon excitation and vertical exciton binding energy, which was found for the monomers, is studied to see whether or not it also holds for the trimers. It appears that $\Delta \mu_{\text {ge }}$ does not correlate with the vertical exciton binding energy for all trimers. An increase in $\Delta \mu_{\text {ge }}$ and a corresponding decrease in vertical exciton binding energy are found only for trimers 8, 10 and 11 compared to the reference 7 (Fig. 11). For trimer 9, a large increase in $\Delta \mu_{\text {ge }}$ and a very small increase in vertical exciton binding energy are found. For the $\mathrm{CH}_{3} / \mathrm{F}$ substituted trimer 12, no significant changes in $\Delta \mu_{\text {ge }}$ and in vertical exciton binding energy are found compared to the reference 7. Only mesomeric groups significantly influence the formation of more separated excitons leading to an increase in $\Delta \mu_{\mathrm{ge}}$. 


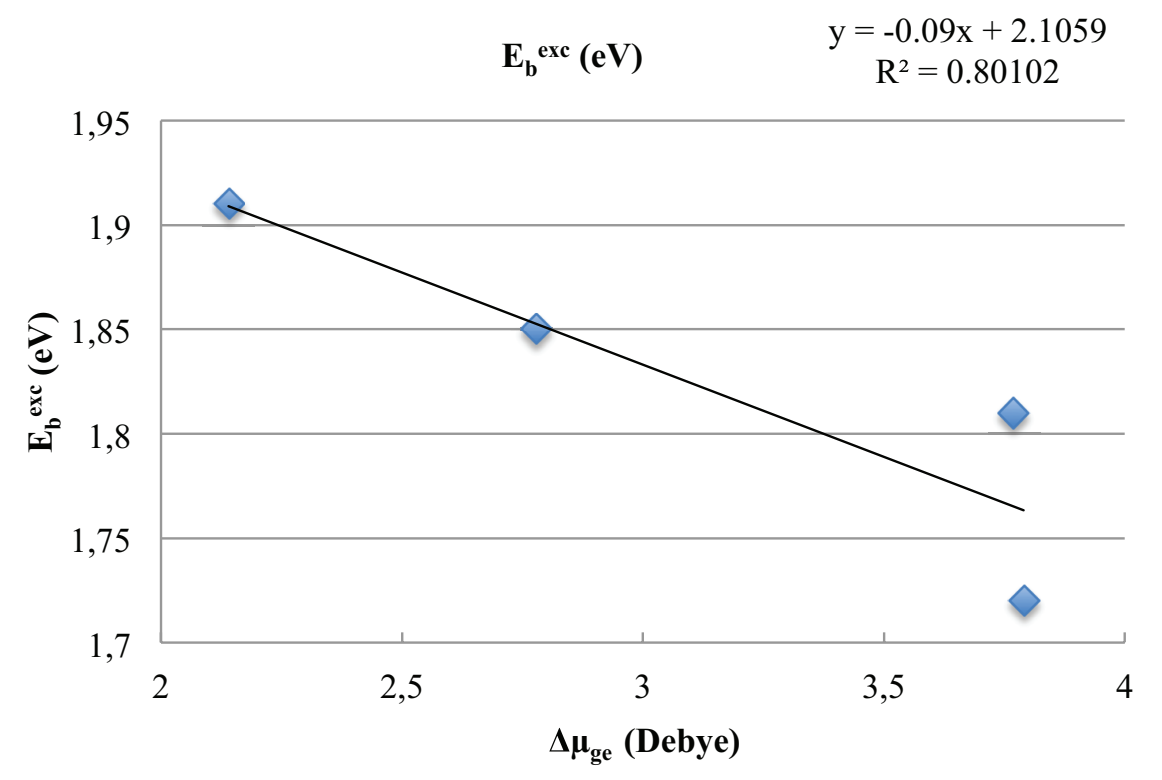

Fig. 11. The vertical exciton binding energy $\left(\mathrm{E}_{\mathrm{b}}{ }^{\mathrm{exc}}, \mathrm{eV}\right)$ as a function of the change in dipole moment upon excitation $\left(\Delta \mu_{\mathrm{ge}}\right.$, Debye). A linear trend line is added together with $\mathrm{R}^{2}$.

\section{Summary and conclusions}

In this work, the influence of push-pull group substitution patterns on excited state properties, in particular the exciton binding energy, of several donor-acceptor co-monomers and their trimers is studied by performing (TD)-DFT (BHandH/6-31G**) calculations. The aim is to understand and engineer the photo-physical properties of new molecular structures. Part of this work focuses on OPV applications to demonstrate practical use of the structure-property relations.

The influence of push-pull group substitution patterns on excited state properties of several monomers can be summarized as follows. A decrease is found in the vertical excitation energy, the bandgap (mostly caused by a decrease in the LUMO energy), and the oscillator strength, with the largest decrease for functional groups that exert mesomeric effects. A good correspondence between the HOMO energy and the vertical ionization potential, and the LUMO energy and the vertical electron affinity is found for all substituted molecules. The only clear distinction between linear- and cross-conjugated substitution patterns is found for the LUMO energy (and vertical electron affinity): the LUMO energy is higher (and the vertical electron affinity is smaller) in the case of linearconjugated substitution patterns.

The influence of push-pull group substitution patterns on properties relevant for OPV applications of several monomers can be summarized as follows. A decrease is found in the electron-hole interaction calculated as $\left[\mathrm{J}_{\mathrm{HL}}-2 \mathrm{~K}_{\mathrm{HL}}\right]$ for the $\mathrm{CCM}$, an increase for the $\mathrm{LCM}$, and a small decrease for substitution with functional groups that exert inductive effects. For $\mathrm{NH}_{2} / \mathrm{NO}_{2}$ substituted molecules, the vertical exciton binding energy decreases (except for 3), with the largest decrease for the CCM. For the $\mathrm{CH}_{3} / \mathrm{F}$ substituted molecule, no significant difference is found compared to the reference molecule. From linear- to cross-conjugation, a reasonably similar decrease is found in $\left[\mathrm{J}_{\mathrm{HL}}-2 \mathrm{~K}_{\mathrm{HL}}\right]$ and the vertical exciton binding energy. The dipole moment change upon excitation becomes larger when the monomer is substituted with functional groups that exert inductive or mesomeric effects (except for 3), with the largest increase for the CCM.

The clear difference in linear- and cross-conjugated push-pull group substitution patterns for the LUMO energy, the vertical electron affinity, the electron-hole interaction calculated as $\left[\mathrm{J}_{\mathrm{HL}}-2 \mathrm{~K}_{\mathrm{HL}}\right]$, 
the quantity $\Lambda$, the vertical exciton binding energy, and the dipole moment change upon excitation, can be understood from simple Hückel theory. For odd alternating hydrocarbons substituted with a by resonance donating group, this theory gives a NBMO with small electron densities at the meta positions. So cross-conjugated push-pull group substitution patterns lead to less $\pi$-delocalised frontier MOs over separate parts of the molecule, whereas linear-conjugated push-pull group substitution patterns lead to more $\pi$-delocalised frontier MOs over similar parts of the molecule.

The computed trends give strong support for a linear relation $\left(\mathrm{R}^{2}: 0.98\right)$ between $\left[\mathrm{J}_{\mathrm{HL}}-2 \mathrm{~K}_{\mathrm{HL}}\right]$ and the observable $\Delta \mu_{\text {ge }}$. A linear relation between $\Delta \mu_{\text {ge }}$ and the vertical exciton binding energy is also found, although with a smaller $\mathrm{R}^{2}$ value $(0.80)$. Still, these results hint to a possible correlation between properties related to chemical composition (i.e., $\left[\mathrm{J}_{\mathrm{HL}}-2 \mathrm{~K}_{\mathrm{HL}}\right]$ and $\Delta \mu_{\mathrm{ge}}$ ) and the vertical exciton binding energy. The overlap density between the frontier MOs alone already reveals valuable information about the relative size of the electron-hole interaction and the vertical exciton binding energy.

When the excited state properties of the monomers are compared to the ones of their trimers, it appears that most of the trends for the monomers still hold, except for the ones that gave a difference between linear- and cross-conjugated substitution patterns because for the trimers this distinction in conjugation pattern does not hold anymore. In general the relative differences are diminished and the effects are usually smaller. Many deviations of the trends for the monomers are found for the trimer with functional groups that exert inductive effects: the vertical excitation energy increases and significant effects of the $\mathrm{CH}_{3} / \mathrm{F}$ groups are found on the HOMO/LUMO energies, the vertical ionisation potential and the vertical electron affinity. For trimers in general the vertical excitation energy decreases by about $0.4-0.8 \mathrm{eV}$, the bandgap decreases by about $0.5-0.6 \mathrm{eV}$ (except for 7: 0.9 $\mathrm{eV}$ ), the LUMO energy decreases by about $0.2-0.4 \mathrm{eV}$ (the vertical electron affinity increases by about 0.6-0.8 eV), and the HOMO energy increases by about $0.3-0.4 \mathrm{eV}$ (the vertical ionisation potential decreases by about $0.6-0.8 \mathrm{eV}$ ), compared to the monomers.

When the properties relevant for OPV applications of the monomers are compared to the ones of their trimers, it appears that for the trimers the vertical exciton binding energy and the change in dipole moment upon excitation are smaller, and the quantity $\Lambda$ is larger. The computed trend in vertical exciton binding energy for the monomers holds reasonably for the trimers. The largest decrease is found for the same type of trimer as the monomer, but this decrease is slightly diminished $(0.2 \mathrm{eV}$ for the trimers compared to $0.3 \mathrm{eV}$ for the monomer). Concerning the change in dipole moment upon excitation, a larger increase is found for the $\mathrm{NH}_{2} / \mathrm{NO}_{2}$ substituted trimers.

Finally, the linear relation between $\Delta \mu_{\text {ge }}$ and the vertical exciton binding energy does not hold for all trimers. Based on the small change in $\Delta \mu_{\mathrm{ge}}$ and no significant change in vertical exciton binding energy for the $\mathrm{CH}_{3} / \mathrm{F}$ substituted trimer, it can be concluded that only mesomeric groups influence the formation of more separated excitons and that this would lead to an increase in dipole moment change upon excitation. Therefore the direct relation found by Carsten et al. [8] between device efficiency and $\Delta \mu_{\mathrm{ge}}$ of a donor-acceptor co-monomer cannot be generalised to oligomers. Whether or not this relation still applies to trimers depends on aspects like the choice of functional groups (i.e., groups that exert inductive or mesomeric effects) and on differences in electron density transfer between the monomer and its extended form (e.g., its trimer), as is shown in this work.

Although the computed trend between $\Delta \mu_{\text {ge }}$ and the vertical exciton binding energy properties does not hold for all trimers, this work indicates that important photo-physical properties, like the exciton binding energy for OPV materials, can be engineered by chemical modification through adding particular functional groups. Application of our findings in the context of organic photovoltaics results in significant support for cross-conjugated mesomeric push-pull group pathways in order to spatially separate the HOMO and LUMO. This substitution pattern possibly leads to lower exciton binding 
energies, without the adverse effect of smaller oscillator strengths compared to linear-conjugated pathways.

\section{Acknowledgements}

Prof. dr. J. C. Hummelen of the University of Groningen is thanked for stimulating discussions. R.W.A.H. acknowledges the Zernike Institute for Advanced Materials ("Dieptestrategie" program) for financial support. This work is part of the research programme of the Foundation of Fundamental Research on Matter (FOM), which is part of the Netherlands Organisation for Scientific Research (NWO). This is a publication of the FOM-focus Group 'Next Generation Organic Photovoltaics', participating in the Dutch Institute for Fundamental Energy Research (DIFFER). The work was partially completed with computing time at the Dutch National Supercomputer Cartesius (SURFsara, SH-213-13).

\section{Appendix A. Supplementary material}

Supplementary data associated with this article can be found, in the online version, at ...

\section{References}

[1] S.R. Forrest, The path to ubiquitous and low-cost organic electronic appliances on plastic, Nature, 428 (2004) 911-918.

[2] M.A. Green, K. Emery, Y. Hishikawa, W. Warta, E.D. Dunlop, Solar cell efficiency tables (version 41), Progr. Photovolt: Res. Appl., 21 (2013) 1-11.

[3] C. Piliego, M.A. Loi, Charge transfer state in highly efficient polymer-fullerene bulk heterojunction solar cells, J. Mater. Chem., 22 (2012) 4141-4150.

[4] G. Yu, J. Gao, J.C. Hummelen, F. Wudl, A.J. Heeger, Polymer photovoltaic cells: enhanced efficiencies via a network of international donor-acceptor heterojunctions, Science, 270 (1995) 17891791.

[5] J.-L. Brédas, J.E. Norton, J. Cornil, V. Coropceanu, Molecular understanding of organic solar cells: the challenges, Acc. Chem. Res., 42 (2009) 1691-1699.

[6] C. Deibel, T. Strobel, V. Dyakonov, Role of charge transfer state in organic donor-acceptor solar cells, Adv. Mater., 22 (2010) 4097-4111.

[7] T.M. Clarke, J.R. Durrant, Charge photogeneration in organic solar cells, Chem. Rev., 110 (2010) 6736-6767.

[8] B. Carsten, J.M. Szarko, L. Lu, H.J. Son, F. He, Y.Y. Botros, L.X. Chen, L. Yu, Mediating solar cell performance by controlling the internal dipole change in organic photovoltaic polymers, Macromolecules, 45 (2012) 6390-6395.

[9] B. Carsten, J.M. Szarko, H.J. Son, W. Wang, L. Lu, F. He, B.S. Rolczynski, S.J. Lou, L.X. Chen, L. Yu, Examining the effect of the dipole moment on charge separation in donor-acceptor polymers for organic photovoltaic applications, J. Am. Chem. Soc., 133 (2011) 20468-20475.

[10] B.S. Rolczynski, J.M. Szarko, H.J. Son, Y. Liang, L. Yu, L.X. Chen, Ultrafast intramolecular exciton splitting dynamics in isolated low-band-gap polymers and their implications in photovoltaic materials design, J. Am. Chem. Soc., 134 (2012) 4142-4152.

[11] R. Tautz, E. Da Como, T. Limmer, J. Feldmann, H.-J. Egelhaaf, E. Von Hauff, V. Lemaur, D. Beljonne, S. Yilmaz, I. Dumsch, S. Allard, U. Scherf, Structural correlations in the generation of polaron pairs in low-bandgap polymers for photovoltaics, Nature Communications, 3:970 (2012) 1-8. 
[12] P.-L.T. Boudreault, A. Najari, M. Leclerc, Processable low-bandgap polymers for photovoltaic applications, Chem. Mater., 23 (2010) 456-469.

[13] H. Valkenier, C.M. Guédon, T. Markussen, K.S. Thygesen, S.J. Van der Molen, J.C. Hummelen, Cross-conjugation and quantum interference: a general consideration?, Phys. Chem. Chem. Phys., (2013).

[14] K.P.C. Vollhardt, N.E. Schore, Organic Chemistry: structure and function, fifth ed., W. H. Freeman and Company New York, 2007.

[15] A.D. Becke, A new mixing of Hartree-Fock and local density-functional theories, J. Phys. Chem., 98 (1993) 1372-1377.

[16] K. Aidas, C. Angeli, K.L. Bak, V. Bakken, R. Bast, L. Boman, O. Christiansen, R. Cimiraglia, S. Coriani, P. Dahle, E. K. Dalskov, U. Ekström, T. Enevoldsen, J. J. Eriksen, P. Ettenhuber, B. Fernández, L. Ferrighi, H. Fliegl, L. Frediani, K. Hald, A. Halkier, C. Hättig, H. Heiberg, T. Helgaker, A. C. Hennum, H. Hettema, E. Hjertenaes, S. Høst, I.-M. Høyvik, M. F. Iozzi, B. Jansík, H. J. A. Jensen, D. Jonsson, P. Jørgensen, J. Kauczor, S. Kirpekar, T. Kjaergaard, W. Klopper, S. Knecht, R. Kobayashi, H. Koch, J. Kongsted, A. Krapp, K. Kristensen, A. Ligabue, O. B. Lutnaes, J. I. Melo, K. V. Mikkelsen, R. H. Myhre, C. Neiss, C. B. Nielsen, P. Norman, J. Olsen, J. M. H. Olsen, A. Osted, M. J. Packer, F. Pawlowski, T. B. Pedersen, P. F. Provasi, S. Reine, Z. Rinkevicius, T. A. Ruden, K. Ruud, V. V. Rybkin, P. Sałek, C. C. M. Samson, A. S. de Merás, T. Saue, S. P. A. Sauer, B. Schimmelpfennig, K. Sneskov, A. H. Steindal, K. O. SylvesterHvid, P. R. Taylor, A. M. Teale, E. I. Tellgren, D. P. Tew, A. J. Thorvaldsen, L. Thøgersen, O. Vahtras, M. A. Watson, D. J. D. Wilson, M. Ziolkowskiand, H. Ågren, The Dalton quantum chemistry program system, Wiley Interdisciplinary Reviews: Computational Molecular Science, (2013) doi: 10.1002/wcms.1172.

[17] M.F. Guest, I.J. Bush, H.J.J. Dam van, P. Sherwood, J.M.H. Thomas, J.H. Lenthe van, R.W.A. Havenith, J. Kendrick, The GAMESS-UK electronic structure package: algorithms, developments and applications, Mol. Phys., 103 (2005) 719-747.

[18] G. Sini, J.S. Sears, J.-L. Brédas, Evaluating the performance of DFT functionals in assessing the interaction energy and ground-state charge transfer of donor/acceptor complexes: tetrathiafulvalenetetracyanoquinodimethane (TTF-TCNQ) as a model case, J. Chem. Theory Comput., 7 (2010) 602609.

[19] TURBOMOLE V6.4 2012, a development of University of Karlsruhe and Forschungszentrum Karlsruhe GmbH, 1989-2007, TURBOMOLE GmbH, since 2007; available from http://www.turbomole.com.

[20] O. Christiansen, H. Koch, P. Jörgensen, The second-order approximate coupled cluster singles and doubles model CC2, Chem. Phys. Lett., 243 (1995) 409-418.

[21] C. Hättig, A. Hellweg, A. Köhn, Distributed memory parallel implementation of energies and gradients for second-order Møller-Plesset perturbation theory with the resolution-of-the-identity approximation, Phys. Chem. Chem. Phys., 8 (2006) 1159-1169.

[22] C. Hättig, F. Weigend, CC2 excitation energy calculations on large molecules using the resolution of the identity approximation, J. Chem. Phys., 113 (2000) 5154-5161.

[23] F. Weigend, M. Häser, H. Patzelt, R. Ahlrichs, RI-MP2: optimized auxiliary basis sets and demonstration of efficiency, Chem. Phys. Lett., 294 (1998) 143-152.

[24] M.J. Peach, P. Benfield, T. Helgaker, D.J. Tozer, Excitation energies in density functional theory: an evaluation and a diagnostic test, J. Chem. Phys., 128 (2008) 044118.

[25] F. Etzold, I.A. Howard, N. Forler, D.M. Cho, M. Meister, H. Mangold, J. Shu, M.R. Hansen, K. Müllen, F. Laquai, The effect of solvent additives on morphology and excited-state dynamics in PCPDTBT:PCBM photovoltaic blends, J. Am. Chem. Soc., 134 (2012) 10569-10583.

[26] I.-W. Hwang, C. Soci, D. Moses, Z. Zhu, D. Waller, R. Gaudiana, C.J. Brabec, A.J. Heeger, Ultrafast electron transfer and decay dynamics in a small band gap heterojunction material, Adv. Mater., 19 (2007) 2307-2312.

[27] O. Vahtras, H. Ågren, P. Jørgensen, H.J.A. Jensen, T. Helgaker, J. Olsen, Multiconfigurational quadratic response functions for singlet and triplet perturbations: the phosphoresence lifetime of formaldehyde, J. Chem. Phys., 97 (1992) 9178-9187.

[28] H. Hettema, H.J.A. Jensen, P. Jørgensen, J. Olsen, Quadratic response functions for a multiconfigurational self-consistent field wave-function, J. Phys. Chem., 97 (1992) 1174-1190. 
[29] H. Ågren, O. Vahtras, H. Koch, P. Jørgensen, T. Helgaker, Direct atomic orbital based selfconsistent-field calculations of nonlinear molecular properties - application to the frequencydependent hyperpolarizability of para-nitroaniline, J. Phys. Chem., 98 (1993).

[30] J. Michl, E.W. Thulstrup, Why is azulene blue and anthracene white? A simple MO picture, Tetrahedron, 32 (1976) 205-209.

[31] A. Szabo, N.S. Ostlund, Modern Quantum Chemistry: Introduction to Advanced Electronic Structure Theory, first revised ed., McGraw-Hill Publishing Company New York, 1989.

[32] C.A. Van Walree, J.H. Lenthe van, B.C. Van der Wiel, On the UV spectrum of cross-conjugated 2,3-diphenyl-1,3-butadiene, Chem. Phys. Lett., 528 (2012) 29-33.

[33] E.J. Baerends, O.V. Gritsenko, R. Van Meer, The Kohn-Sham gap, the fundamental gap and the optical gap: the physical meaning of occupied and virtual Kohn-Sham orbital energies, Phys. Chem. Chem. Phys., 15 (2013) 16408-16425.

[34] E. Hückel, Quantentheoretische Beiträge zum Benzolproblem., Zeitschrift für Physik, 70 (1931) 204-286.

[35] M. Klokkenburg, M. Lutz, A.L. Spek, J.H. Van der Maas, C.A. Van Walree, Electron delocalisation in cross-conjugated $p$-phenylenevinylidene oligomers, Chem. Eur. J., 9 (2003) 35443554 . 